\title{
Effect of carbamazepine on the single oral dose pharmacokinetics of perospirone and its active metabolite
}

Takuya Masui $^{a^{*}}$, Ichiro Kusumi ${ }^{\mathrm{a}}$, Yoshito Takahashi ${ }^{\mathrm{b}}$, and Tsukasa Koyama ${ }^{\mathrm{a}}$

a Department of Psychiatry, Hokkaido University Graduate School of Medicine. Kita 15

Nishi 7, Kita-ku, Sapporo, Hokkaido, Japan.

b Department of Neuropsychiatry, Sapporo Municipal General Hospital (Seiryoin). 4-18,

Toyohira-ku, Sapporo, Hokkaido, Japan.

Correspondence: Takuya Masui, M.D.

Department of Psychiatry, Hokkaido University Graduate School of Medicine

North 15, West 7, Sapporo 060-8638, Japan

TEL: +81-11-716-1161 ext.5973

FAX: +81-11-706-5081

E-mail: masui@med.hokudai.ac.jp 


\section{Abstract}

Perospirone is a serotonin 5- $\mathrm{HT}_{2 \mathrm{~A}}$ and dopamine $\mathrm{D}_{2}$ receptor antagonist originated in Japan. It has been considered that perospirone is metabolized to ID-15036 mainly by CYP3A4 based on an in vitro study. To investigate the metabolism of perospirone in humans, the authors measured the concentration of perospirone and ID-15036 after single oral dose of perospirone $(8 \mathrm{mg})$ in 10 healthy male subjects, before and during coadministration of carbamazepine, known as a potent inducer of CYP3A4. Before carbamazepine coadministration, the peak plasma concentrations \pm SD of perospirone and ID-15036 were $4.0 \pm 4.3 \mathrm{ng} / \mathrm{ml}$ and $11.7 \pm 7.1 \mathrm{ng} / \mathrm{ml}$, respectively. During carbamazepine coadministration, the concentration of perospirone was decreased lower than detection limit, and that of ID-15036 was $6.0 \pm 1.7 \mathrm{ng} / \mathrm{ml}$. The concentrations of perospirone and ID-15036 were influenced significantly by the treatment with carbamazepine, and this was probably attributable to the induction of CYP3A4. This study provided an in vivo evidence of involvement of CYP3A4 in the metabolism of perospirone.

\section{Key words:}

Atypical antipsychotics, Carbamazepine, Healthy subject, Perospirone, Pharmacokinetics, Schizophrenia

Abbreviations: CBZ: Carbamazepine

CYP: Cytochrome P450

EPS: Extra-pyramidal symptoms

PER: Perospirone 


\section{Introduction}

Perospirone (PER) is a serotonin 5- $\mathrm{HT}_{2 \mathrm{~A}}$ and dopamine $\mathrm{D}_{2}$ receptor antagonist originated in Japan. This agent has become widely used because of its fewer side effect profile than conventional antipsychotics. In the clinical situation, the risk of side effect such as extra-pyramidal symptoms (EPS) induced by PER is likely to increase in proportion to its dose. It is believed that EPS is related to some serotonin/dopamine interaction (Casey, 1996). Our previous reports are also supportive to this observation (Takahashi et al., 1998), in which PER predominantly showed a higher $5-\mathrm{HT}_{2 \mathrm{~A}} / \mathrm{D}_{2}$ ratio at lower doses, while the advantage of 5- $\mathrm{HT}_{2 \mathrm{~A}}$ receptor occupancy over $\mathrm{D}_{2}$ receptor occupancy was lost at higher doses. Such a tendency is very similar to risperidone (Matsubara et al., 1993), and it has been reported that the incidence of EPS was significantly higher in patients treated with higher dose of risperidone (Marder and Meibach, 1994). On the other hand, 5- $\mathrm{HT}_{2 \mathrm{~A}}$ receptor occupancy by ID-15036, a major active metabolite of PER, was predominantly observed even at higher doses. This suggests that PER has a biochemical profile similar to that for clozapine and olanzapine (Takahashi et al., 1998). Consequently, it is assumed that clinical characteristic of ID-15036 is different from that of PER.

In previous case series, we presented that carbamazepine (CBZ) was effective against perospirone-induced akathisia (Masui et al., 2005). It has been considered that PER is metabolized to ID-15036 mainly by cytochrome P450 (CYP) 3A4 based on an in vitro study (Mizuno, et al., 2003). Therefore, when PER is administered with CBZ, well known as a potent inducer of CYP3A4 (Wilkinson, 1996), it is expected that pharmacokinetics of PER can be altered. And this pharmacokinetic alteration may 
be the main mechanism underlying anti-akathisic effect of CBZ.

Here, the authors investigated the pharmacokinetics of PER at single oral dose under concomitant use of

CBZ, and the in-vivo effect of altered pharmacokinetics by a measurement of prolactin levels, which is regarded as a indicator of dopamine $\mathrm{D}_{2}$ receptor blockade, in healthy volunteers.

\section{Methods}

Subjects

The subjects of this study were 10 physically and mentally healthy male volunteers. The mean \pm S.D.(range) of age and body weight were $37.6 \pm 8.8(28-53)$ years old and $71.6 \pm 9.3(57-86) \mathrm{kg}$. None had taken any drug or food that affects CYP $3 \mathrm{~A} 4$ activity for at least 4weeks before introduction of this study. After complete explanation of the study, the written informed consent was obtained from all subjects. The study protocol was approved by the Ethics Committee of Hokkaido University Graduate School of Medicine.

Protocol

This study was conducted in an open design. After an overnight fasting, subjects ate light meal at 8:30 am, and took $8 \mathrm{mg}$ tablet of PER (Lullan®, Sumitomo Pharmaceuticals, Osaka, Japan) orally with a cup of tap water at 9 am of the $1^{\text {st }}$ day. No food was allowed until $4 \mathrm{hr}$ after PER dosing. Blood samples $(5 \mathrm{ml}$ each) were taken just before and at $0.5,1,1.5,2,3,4,5,6,8$ and $10 \mathrm{hr}$ after PER dosing, for the 
measurement of drug concentration and prolactin levels. From the $3^{\text {rd }}$ to $7^{\text {th }}$ day, two $100 \mathrm{mg}$ tablets of CBZ (telesmin ${ }^{\circledR}$, Mitsubishi Pharma Corporation, Osaka, Japan) were given orally at $9 \mathrm{pm}$. After an overnight fasting and light meal at 8:30 am on the $8^{\text {th }}$ day, PER dosing and blood sampling were done in the same schedule as the $1^{\text {st }}$ day.

Assay

Plasma concentrations of PER and ID-15036 were measured by the high-performance liquid chromatography (HPLC) method reported by Yasui-Furukori et al. (2003). The lowest limits of detection and quantification were 0.1 and $0.2 \mathrm{ng} / \mathrm{mL}$ for both perospirone and ID-15036. And the values of the intra-day relative standard deviations were less than $3.4 \%$ for PER, and 3.7\% for ID-15036, in the concentration range of $0.3-30 \mathrm{ng} / \mathrm{mL}$. Plasma concentration of CBZ was determined using HPLC with minor modification (Hikida et al., 1989). The lowest limits of detection and quantification were 0.05 and $0.10 \mu \mathrm{g} / \mathrm{mL}$, respectively.

Plasma prolactin levels were also measured with enzyme immunoassay methods using commercial kit (IMx ${ }^{\circledR}$ Prolactin, Abbott Japan, Tokyo, Japan).

Statistical analyses

The paired t-test was used for the comparison of the plasma drug concentrations, plasma prolactin levels and pharmacokinetic parameters before and during CBZ coadministration. A P value of 0.05 or less was regarded as significant. 


\section{Results}

The mean plasma concentrations of PER and ID-15036 before and during CBZ coadministration are shown in Figure 1. Before CBZ coadministration, the plasma concentration of PER was detectable up to 8 hours after dosing. During CBZ coadministration, the plasma concentration of PER decreased under lowest limit of detection at all points of blood sampling in 6 subjects, and the peak plasma PER concentrations in the remaining 4 subjects also decreased $(<1.9 \mathrm{ng} / \mathrm{ml}$. Data not shown.). ID-15036 also decreased but was detectable up to 5 hours in all subjects during CBZ coadministration.

Table 1 shows the pharmacokinetic parameters of PER and ID-15036, the plasma concentrations of CBZ at PER dosing and the peak plasma prolactin levels after dosing. Because the plasma concentration of PER was undetectable in 6 subjects $(60 \%)$ during $\mathrm{CBZ}$ coadministration, their pharmacokinetic parameters were not calculated. $\mathrm{CBZ}$ significantly $(\mathrm{P}<0.05$ to 0.01$)$ decreased mean peak plasma concentration $\left(\mathrm{C}_{\max }\right)$, elimination half-life $\left(\mathrm{T}_{1 / 2}\right)$, area under the plasma concentration-time curve (AUC) from 0 to 10 hours $\left(\mathrm{AUC}_{0-10 \mathrm{~h}}\right)$ and $\mathrm{AUC}$ from 0 hour to infinity $\left(\mathrm{AUC}_{0-\infty}\right)$ of ID-15036.

The peak plasma prolactin level significantly $(\mathrm{P}<0.01)$ decreased during $\mathrm{CBZ}$ coadministration.

\section{Discussion}

In the present study, $\mathrm{C}_{\max }, \mathrm{T}_{1 / 2}$ and $\mathrm{AUC}_{0-\infty}$ of ID-15036 were 3-fold, 1.5 -fold and 5-fold higher than 
those of PER before CBZ coadministration. Similar predominancy of ID-15036 over PER were shown in clinical phase I trial of PER (Inanaga et al., 1997). Based on these results and our previous receptor binding study (Takahashi et al., 1998), ID-15036 is expected to have important roles regarding both antipsychotic effect and amelioration of EPS when PER is administered orally, although behavioral pharmacology of ID-15036 has not been studied.

During CBZ coadministration, pharmacokinetics of PER and ID-15036 were significantly influenced. To our knowledge, this is the first in-vivo evidence indicating that CBZ influenced the pharmacokinetics of PER. Decreased absorption and/or accelerated metabolism were considered as causes of these altered pharmacokinetics. But it is more likely that induced metabolism by CBZ is the predominant factor at least for the altered ID-15036 kinetics, considering $\mathrm{T}_{1 / 2}$ of ID-15036 was shortened from 2.2 to 1.2 hours. CBZ is regarded as a potent inducer of CYP3A4, therefore, it is possible that metabolism of PER was also accelerated by CBZ. This possibility is consistent with a previous report showing that PER is metabolized mainly by CYP3A4 (Mizuno et al., 2003). However, it is hardly expected that PER and ID-15036 are catalyzed by only CYP3A4. Although plasma concentrations of these two chemicals were reduced during CBZ coadministration, the change in ID-15036 concentration was less prominent than that in PER concentration. Therefore, some other enzymes are considered to be involved in the metabolic pathway of, at least, ID-15036.

Peripheral prolactin level has been used as a sensitive indicator of pituitary dopamine $\mathrm{D}_{2}$ receptor blockade (Turrone et al., 2002). During CBZ coadministration, maximum prolactin level was significantly lower than that of before $\mathrm{CBZ}$ coadministration. $\mathrm{CBZ}$ does not decrease prolactin level by itself, therefore, 
this indicates that CBZ reduces PER concentration in central nerve system as shown in peripheral, and that $\mathrm{D}_{2}$ receptor blockade was weakened.

\section{Conclusion}

This study showed that CBZ greatly decreased plasma PER concentration, and confirmed the involvement of CYP3A4 in metabolism of PER in human. Although coadministration of CBZ during PER therapy works successfully in some cases, it is possible that decrease in PER concentration may be at risk of aggravation of psychotic symptoms. Therefore, clinician should pay more attention when PER and CBZ are administered concomitantly.

\section{Acknowledgement}

This work was partly supported by grants-in aid for Scientific Research no.13670978 (I. Kusumi) from Japanese Ministry of Education, Culture, Sports, Science and Technology. 


\section{References}

Casey, D.E., 1996. Extrapyramidal syndromes and new antipsychotic drug: findings in patients and non-human primate models. Br. J. Psychiatry. 168, 32-39.

Hikida, K., Inoue, Y., Kojima, N., 1989. Determination of pranoprofen in serum by automated colum-switching high-performance liquid chromatography. Jpn. J. Clin. Chem. 18180-18183. Inanaga, K., Irie, S., Urae, A., Urae, R., 1997. Phase I study of perospirone HCl (SM-9018). Kiso to Rinsho. 31, 2113-2157.

Marder, S.R., Meibach, R.C., 1994. Risperidone in the treatment of schizophrenia. Am. J. Psychiatry. 151, 825-835.

Masui, T., Kusumi, I., Takahashi, Y., Koyama, T., 2005. Efficacy of carbamazepine against neuroleptic-induced akathisia in treatment with perospirone: case series. Prog Neuropsychopharmacol Biol Psychiatry. 29, 343-346.

Matsubara, S., Matsubara, R., Kusumi, I., Koyama, T., Yamashita, I., 1993. Dopamine D1, D2, and serotonin2 receptor occupation by typical and atypical antipsychotic drugs in vivo. J.

Pharmacol. Exp. Ther. 251, 238-246.

Mizuno, Y., Tani, N., Komuro, S., Kanamaru, H., Nakatsuka, I., 2003. In Vitro metabolism of perospirone in rat, monkey, and human liver microsomes. Eur. J. Drug Metab. Pharmacokinet. $28,59-65$.

Takahashi, Y., Kusumi, I., Ishikane, T., Koyama, T., 1998. In vivo occupation of dopamine D1, 
D2 and serotonin2A receptors by novel antipsychotic drug, SM-9018 and its metabolite, in rat brain. J. Neural Transm. 105, 181-191.

Turrone, P., Kapur, S., Seeman, M.V., Flint, A.J., 2002. Elevation of prolactin levels by atypical antipsychotics. Am. J. Psychiatry. 159, 133-135.

Wilkinson, G.R., 1996. Cytochrome P4503A(CYP3A) metabolism: prediction of in vitro activity in humans. J. Pharmacokinet. Biopharm. 24, 475-490.

Yasui-Furukori, N., Inoue, Y., Tateishi, T., 2003. Determination of a new atypical antipsychotic agent perospirone and its metabolite in human plasma by automated column-switching high-performance liquid chromatography. J. Chromatography B. 789, $239-24$ 


\section{Legends for figure}

Figure 1.

Mean plasma concentrations of perospirone (left) and ID-15036 (right), before (solid symbols) and during (open symbols) carbamazepine coadministration.

Each point represents the mean concentration of 10 subjects (perospirone before carbamazepine, and ID-15036 before and during carbamazepine) or 4 subjects (perospirone during carbamazepine). 


\section{Table 1.}

Each value represents the mean \pm SD of 10 subjects (perospirone before carbamazepine, and ID-15036 before and during carbamazepine) or 4 subjects (perospirone during carbamazepine).

All $\mathrm{P}$ values resulted from the use of paired $\mathrm{t}$ tests (two sided).

Abbreviations: $\mathrm{Cmax}=$ peak plasma concentration; $\mathrm{Tmax}=$ time to $\mathrm{Cmax}$; $\mathrm{T} 1 / 2=$ elimination half-life;

AUC0-10h=area under the plasma concentration-time curve(AUC) from 0 to 10 hours; AUC $0-\infty=$ AUC from 0 hour to infinity; $\mathrm{NC}=$ not calculated; $\mathrm{ND}=$ not detectable; $\mathrm{NS}=$ not significant. 


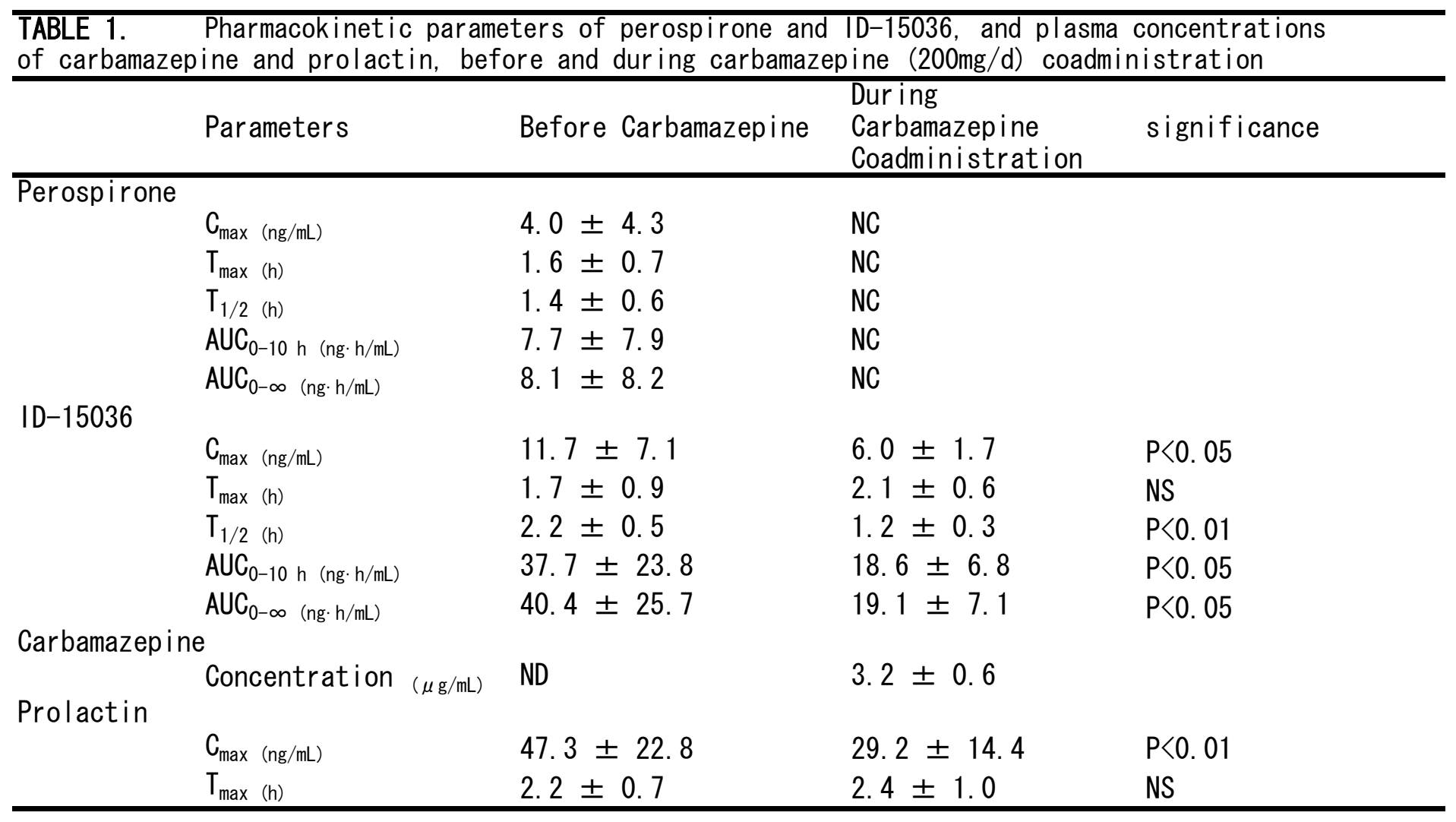

Each value represents the mean \pm SD of 10 subjects (perospirone before carbamazepine, and ID-15036 before and during carbamazepine) or 4 subjects (perospirone during carbamazepine).

All $P$ values resulted from the use of paired $t$ tests (two sided)

Abbreviations: Cmax=peak plasma concentration; Tmax=time to Cmax; $T 1 / 2=e$ limination half-life;

AUC $0-10 h=a r e a$ under the plasma concentration-time curve(AUC) from 0 to 10 hours; AUC0- $\infty=A U C$ from 0 hour to infinity;

$\mathrm{NC}=$ not calculated; $\mathrm{ND}=$ not detectable; $\mathrm{NS}=$ not significant. 

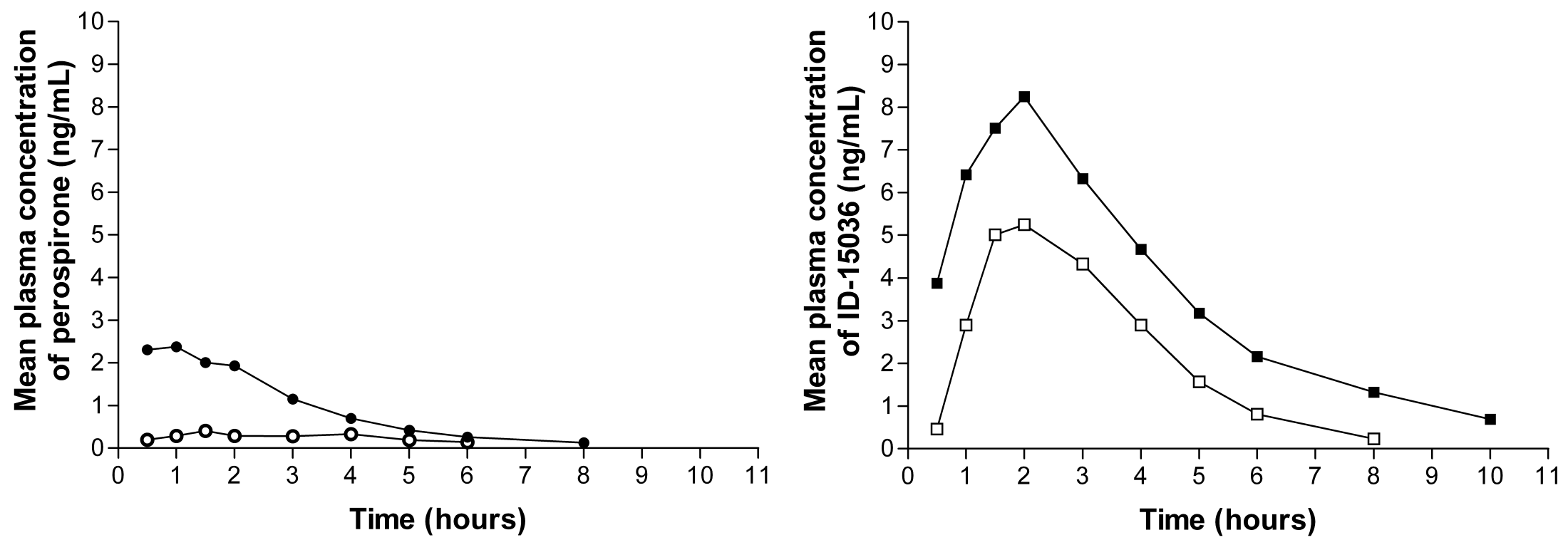